\title{
Experiência-piloto de assistência domiciliar: idosos acamados de uma Unidade Básica de Saúde, Porto Alegre, Brasil
}

\author{
PILOT-EXPERIENCE IN HOME CARE: BEDRIDDEN AGED PATIENTS OF A BASIC \\ HEALTH UNIT, PORTO ALEGRE, BRAZIL
}

\author{
EXPERIENCIA PILOTO DE ASISTENCIA A DOMICILIO: ANCIANOS ENCAMADOS EN \\ UNA UNIDAD BÁSICA DE SALUD, EN PORTO ALEGRE, BRASIL
}

\author{
Giselda Quintana Marques', Ivani Bueno de Almeida Freitas ${ }^{2}$
}

\begin{abstract}
RESUMO
O estudo teve por objetivos: descrever o processo de desenvolvimento do projetopiloto de assistência a idosos acamados, da Unidade Básica de Saúde IAPI; e identificar aspectos demográficos, sociais e de saúde desses idosos, bem como aspectos relevantes relatados pela equipe, na implantação da assistência domiciliar. A pesquisa teve características descritivas e avaliativas. Foram revisadas as fichas cadastrais e os prontuários dos pacientes atendidos, assim como os registros de avaliação do projeto. A experiência-piloto permitiu o desenvolvimento de habilidades na equipe, foi enriquecedora e de grande responsabilidade para profissionais e cuidadores. Apontou para a continuidade da assistência domiciliar, fazendo-se necessários ajustes na sua organização, com a finalidade de ampliar os espaços de assistência e a qualidade do que vinha sendo ofertado à população.
\end{abstract}

\section{DESCRITORES}

Cuidados de enfermagem.

Idoso.

Serviços de Assistência Domiciliar.

\section{ABSTRACT}

The objectives of this study were to describe the development of a pilot-project in home care to bedridden aged patients at a Basic Health Unit, and identify demographic, social and health aspects of these patients, as well as relevant aspects reported by the health team that implemented the home care. The study had descriptive and evaluative characteristics. The patients' enrollment forms and health records and the project's records were analyzed. The pilot-experience permitted to develop the team's skills, in addition to being enriching and of great responsibility for the professionals and caregivers involved. The results indicated the need for continuous home care and adjustments in its organization with the purpose of increasing the areas for health care and improving the population's quality of life.

\section{KEY WORDS}

Nursing care.

Aged.

Home Care Services.

\section{RESUMEN}

El estudio tuvo por objetivos describir: el proceso de desarrollo del proyecto piloto de asistencia a ancianos encamados, de la Unidad Básica de Salud IAPI; identificar aspectos demográficos, sociales y de salud de esos ancianos; $y$, identificar los aspectos relevantes relatados por el equipo, en la implantación de la asistencia a domicilio. La investigación tuvo características descriptivas y de evaluación. Fueron revisadas las fichas de inscripción de los pacientes atendidos, así como los registros de evaluación del proyecto. La experiencia piloto permitió el desarrollo de habilidades en el equipo, fue enriquecedora y de gran responsabilidad para los profesionales y cuidadores. El resultado apuntó para la continuidad de la asistencia a domicilio, siendo necesario realizar ajustes en su organización, con la finalidad de ampliar los espacios de asistencia y la calidad de lo que estaba siendo ofrecido a la población.

\section{DESCRIPTORES}

Atención de enfermería.

Anciano.

Servicios de Atención de Salud a Domicilio.

${ }^{1}$ Doutoranda em Enfermagem da Escola de Enfermagem da Universidade Federal do Rio Grande do Sul. Enfermeira do Centro de Saúde IAPI. Secretaria Municipal da Saúde de Porto Alegre. Porto Alegre, RS, Brasil. gqmarques@terra.com.br ${ }^{2}$ Mestre em Saúde Coletiva. Enfermeira do Centro de Saúde IAPI. Secretaria Municipal da Saúde de Porto Alegre. Professora de Enfermagem da Faculdade de Enfermagem da Universidade Vale do Rio dos Sinos. Porto Alegre, RS, Brasil. ivanifreitas@terra.com.br 


\section{INTRODUÇÃO}

O processo de envelhecimento humano tem sido tema de discussão em quase todos os países do mundo, e, no Brasil, toma proporções alarmantes visto que a estimativa de vida da população tem aumentado significativamente. Isso se deve à melhoria das condições de vida, de saneamento básico, de trabalho, de educação, bem como das condições tecnológicas que possibilitaram que se vivesse mais e com melhor qualidade.

No Brasil, o decréscimo de uma situação de alta fecundidade e alta mortalidade tem propiciado mudanças significativas na sua pirâmide populacional, caracterizada por um aumento progressivo e acentuado da população adulta e idosa. A Organização das Nações Unidas, desde 1982, considera idoso o indivíduo com idade igual ou superior a 60 anos; no Brasil, adotou-se a mesma faixa etária para caracterizar a população idosa(1).

As mudanças na pirâmide populacional acarretaram uma série de consequências sociais, culturais e epidemiológicas, que a sociedade e as Instituições ainda não estão preparadas para enfrentar. A rápida transição nos perfis de saúde, com predomínio das enfermidades crônicas não transmissíveis e pela importância crescente de fatores de risco para a saúde, têm requerido dos profissionais, da sociedade e do Estado, ações preventivas e curativas, em diversos níveis.

Embora envelhecer não signifique diretamente adoecer e estar dependente, sem dúvida indica uma maior fragilidade e vulnerabilidade que aumenta conforme a idade cronológica dos indivíduos é mais avançada, aliada ao contexto social e ambiental em que o idoso se insere. Na maior parte dos casos, a responsabilidade de cuidados ao idoso fragilizado é assumida pela família, que nem sempre está preparada para tal condição. Esse cuidado se concretiza nas ações cotidianas da vida diária e envolve apoio funcional, social, econômico, material e afetivo ${ }^{(2)}$.

O Sistema de Saúde, por meio das Instituições, tem tentado redefinir suas prioridades, estabelecendo estratégias que possam tratar e prevenir doenças que causem incapacidade e buscando formas de atenção à saúde que ampliem a autonomia dos sujeitos e que promovam qualidade de vida à população( ${ }^{(3)}$.

O número de idosos, no Brasil, correspondia a $10 \%$ da população brasileira, em 2006. Na ocasião, esse número era superior a 18 milhões de pessoas, havendo um crescimento de 5 milhões, no período de 10 anos (1995 - 2005). Grande parte da população de idosos localiza-se nas Regiões Sul e Sudeste do Brasil. No Rio Grande do Sul, são 1.330.034 e, em Porto Alegre, 425.066(4).

$\mathrm{O}$ atendimento à saúde de idosos é mais oneroso que o de outras parcelas da população. O valor médio por inter- nação hospitalar (SUS) no período 2000 - 2005 para cuidados prolongados ou crônicos, no Brasil e no Sul do País foi respectivamente da ordem de $\mathrm{R} \$ 4.155,00-\mathrm{R} \$ \mathrm{8.630,00} \mathrm{e}$ $\mathrm{R} \$ 1.430,00-\mathrm{R} \$ 3.124,00^{(5)}$. Em Porto Alegre, em 2007, o custo de internação para o segmento de 1-49 anos, variou de $\mathrm{R} \$ 673,95$ a $\mathrm{R} \$ 958,25$ enquanto para os de 60 anos ou mais, variou de $R \$ 1.025,36$ a $R \$ 1.530,76^{(6)}$.

Uma das estratégias adotada pelos serviços para desonerar o Estado e modificar o modo tradicional de produção em saúde, é a inclusão da atenção domiciliar no rol de modalidades de atendimento, a qual se caracteriza pela visita da equipe de saúde ao domicílio do usuário com o objetivo de avaliar suas necessidades e as de sua família, considerando a disponibilidade do serviço e constando de plano assistencial e orientações. Atendimento domiciliar pressupõe ações complexas, exigindo técnica e periodicidade da equipe de saúde, de acordo com as necessidades evidenciadas $^{(7)}$.

Essa modalidade tem resultado em maior conforto e segurança ao idoso e sua família, bem como proporcionado cuidado humanizado e qualidade de vida, se comparada ao atendimento institucional ${ }^{(8-9)}$. Mas a concreção dessa perspectiva requer um diagnóstico situacional favorecedor de informações quantitativas e qualitativas que contribuam efetivamente para o conhecimento da realidade de saúde da população idosa e da sua dinâmica domiciliar e familiar. As orientações propiciadas por essas informações permitem a tomada de decisões, de forma que o planejamento do serviço de saúde esteja organizado pelo princípio da equidade, priorizando aqueles que mais necessitem de cuidados.

A permanência do idoso no seu domicílio é muito importante para mantê-lo estimulado pela vida, diminuindo as dificuldades e os limites impostos pela idade avançada. Seu bem-estar dependerá de retaguarda familiar. A família continua sendo uma fonte de sustento e cuidado para os idosos, em todo o mundo. A sua disponibilidade em morar junto e assumir o compromisso de cuidar deles é fundamental.

Sendo assim, o cuidado domiciliar tem se tornado um desafio para os familiares cuidadores, doravante denominados cuidadores primários ${ }^{(10)}$, bem como aos serviços de saúde, pois exige, dos primeiros, que assumam a principal responsabilidade pelo idoso e o desempenho da maior parte das tarefas de cuidado, dedicação total, dia e noite e dos serviços de saúde, um redesenho das ações e uma visão para além dos serviços hoje ofertados nas Unidades de Saúde.

Contudo, a implementação de novas intervenções na Área da Saúde requer dos profissionais a adoção de paradigma condizente com as necessidades sociais e de saúde da população, concatenado com os princípios do Sistema 
Único de Saúde e que não se restrinja às ações realizadas nas Unidades de Saúde e com ênfase nos aspectos biológicos.

A necessidade de responder às frequentes demandas de familiares de idosos acamados, moradores da área de atuação da Unidade Básica de Saúde/UBS IAPI que solicitavam visita domiciliar para acompanhamento médico e/ou de enfermagem aos seus idosos, aliada ao fato, de significativa relevância, de a UBS IAPI, localizar-se na segunda região da cidade com maior número de idosos, 11.000 pessoas, fez com que a Coordenação dessa Unidade apresentasse um projeto de atendimento domiciliar a pacientes acamados, que foi composto de 3 etapas $^{(11)}$ : identificar o perfil socioeconômico e os níveis de dependência de idosos fragilizados do território; identificar necessidades comuns e passíveis de serem trabalhadas em diferentes modalidades de atendimento; propor um projeto-piloto de assistência domiciliar e estabelecer protocolos de atendimento.

\section{OBJETIVOS}

Como propósito deste estudo buscou-se descrever o processo de construção e desenvolvimento do projeto-piloto de assistência ao idoso acamado da UBS IAPI, com ênfase nos objetivos específicos:

a) descrever o processo de construção da assistência domiciliar ao idoso acamado;

b) identificar os aspectos demográficos, sociais e de saúde dos idosos assistidos no projeto;

c) identificar aspectos relevantes, relatados pela equipe, na implantação da assistência domiciliar.

\section{MÉTODO}

Este estudo compõe-se de uma fase descritiva e de uma abordagem qualitativa da temática inserida no projeto intitulado: O cuidado no domicílio: um estudo de caso no Município de Porto Alegre, desenvolvido no Programa de Pós-Graduação em Saúde Coletiva pela Universidade Vale do Rio dos Sinos/RS e aprovado sob o número 132 em 06/02/07 pelo Comitê de Ética em Pesquisa da Secretaria Municipal da Saúde de Porto Alegre.

A pesquisa teve características descritivas e avaliativas do processo de construção do atendimento domiciliar a idosos acamados. A pesquisa avaliativa utiliza métodos científicos e pode ser definida como sendo o procedimento que faz um julgamento ex-post de uma intervenção, geralmente com o objetivo de ajudar, na tomada de decisão. Trata-se de avaliar a pertinência, os fundamentos teóricos, a produtividade, os efeitos e as relações que se estabelecem entre a intervenção e o contexto social em que se situa ${ }^{(12)}$.

Avaliaram-se aspectos da organização do trabalho, contemplando as relações entre trabalhadores, famílias, Unidade de Saúde e demais setores envolvidos com o projeto, não sendo contemplada a avaliação clínica dos acamados, por insuficiência de registros nos prontuários.

Utilizaram-se dados obtidos nas fichas cadastrais, nos prontuários dos pacientes atendidos no Projeto-Piloto de Assistência Domiciliar a Idosos Acamados, no período de maio a dezembro de 2003 e nos registros de avaliação desse projeto (dados secundários).

Foram avaliados 25 cadastros de pacientes e 21 prontuários, visto que 4 pacientes não foram acompanhados pelo projeto. Identificaram-se, nos prontuários, idade, sexo, condições da moradia, existência de cômodo só para si e de cuidador familiar ou contratado, grau de dependência para realização de atividades de vida diária e necessidade de acompanhamento sistemático pela equipe de saúde, bem como as condições domiciliares para o cuidado do doente. Identificaram-se, também, as principais intervenções de enfermagem requeridas pela família. $O$ instrumento de avaliação constava de questões abertas e fechadas. Também foram avaliados os registros de evoluções dos profissionais.

Os documentos institucionais serviram de apoio, abrangendo as resoluções do setor Saúde, projeto de atendimento domiciliar, incluindo protocolo e planilhas.

O estudo não ofereceu risco aos participantes, pois Ihes foi preservado o anonimato e o caráter confidencial das informações contidas nas fichas cadastrais e nos prontuários, obedecendo aos aspectos éticos do Conselho Nacional de Saúde, estabelecidos na Resolução $196 / 96^{(13)}$.

\section{RESULTADOS}

\section{Descrição do processo de construção da assistência domiciliar ao idoso acamado na UBS/IAPI}

O processo de construção da Assistência Domiciliar requereu a realização de uma pesquisa diagnóstica denominada Caracterização de Idosos de uma área adscrita a uma Unidade de Saúde: aspectos socioeconômicos e níveis de dependência para o cuidado ${ }^{(14)}$ pela qual foram identificadas as necessidades dos acamados e estabelecidas as prioridades e as diferentes modalidades de atendimento domiciliar.

A partir disso construiu-se o projeto-piloto com duração de um ano, de cuja elaboração e execução participaram profissionais enfermeiros, fisioterapeuta e fonoaudióloga. Ainda participaram da assistência domiciliar, alunos de cursos de Graduação em Enfermagem de Escolas da Região Metropolitana e da Capital, na disciplina de Estágio Curricular II.

A partir da avaliação e da discussão da experiência(15) 0 projeto-piloto subsidiou a instalação de um programa que passou a atender pessoas acamadas de todas as faixas etárias e que recebeu o nome de Programa de Atendimento Domiciliar ao Acamado (PADA), em 2005. 


\section{Organização da infra-estrutura administrativa para a assistência domiciliar ao idoso}

Para início das atividades do projeto foi montada uma sala na área física do Serviço do Idoso, da Unidade, onde foram organizados os cadastros, as planilhas de atendimento, os arquivos, os prontuários e os materiais para a assistência. Lá também eram realizadas as reuniões semanais para discussão de casos e ajustes do protocolo de atendimento domiciliar. Definiu-se uma coordenadora do projeto, que no primeiro momento foi a fonoaudióloga, com a participação direta da Coordenação do Serviço de Enfermagem que organizou, com sua equipe, os materiais e insumos, em maletas de atendimento, assim como as escalas de atendimento de enfermagem.

Foi elaborado um instrumento para o cadastro de idosos que solicitassem atendimento; esse cadastro era avaliado pela Coordenadora do Projeto que o encaminhava para discussão em reunião, onde eram estabelecidas as equipes de atendimento. Os profissionais realizavam as visitas, prioritariamente em duplas. Nas avaliações iniciais procurou-se colocar profissionais de diferentes categorias funcionais para diversificar o olhar/ escuta e o estabelecimento de prioridades de atendimento. Após a visita era preenchido um documento onde o avaliador estabelecia as prioridades e o tipo de acompanhamento necessário. Em caso de dispensa de acompanhamento domiciliar, o familiar era orientado, no próprio domicílio, sobre os critérios do projeto e os motivos da não aceitação, sendo registrado, no cadastro, o motivo da dispensa.

Os critérios para inclusão de idosos foram: ter 60 anos ou mais, estar acamado, morar no território do entorno à Unidade (em um perímetro estabelecido, de mais ou menos 20 minutos de caminhada), de preferência que não necessitasse o uso de transporte, possuir vínculo com profissional médico para o tratamento, não estar recebendo terapia endovenosa, e contar com a presença de cuidador que se responsabilizasse pelo contrato de assistência. Os critérios de desligamento eram: ausência de cuidador, descumprimento do contrato de cuidados construído no plano de atendimento domiciliar, condição clínica do paciente que fosse condizente com o deslocamento até a Unidade de Saúde, mudança de domicílio que estivesse fora da área de atuação da UBS, ausência de condições domiciliares que promovessem a assistência, tivesse alta e incorresse em óbito(16).

Os materiais foram organizados em maletas, por tipo de atendimento (sondagem vesical, sondagem nasoenteral, curativos, visita clínica, etc); cada profissional era responsável por conferir os materiais e insumos e fazer a reposição, ao término do atendimento. $O$ registro das visitas e do atendimento prestado era feito em prontuário, conforme a abordagem de cada profissional e no impresso do Sistema de Informações Ambulatoriais do Sistema Único de Saúde (SIA/SUS). Os prontuários eram organizados, após a visita de avaliação inicial, e guardados em arquivo, com organização alfanumérica, e conforme os idosos eram cadastrados no Projeto.

Como método de organização para os atendimentos foi utilizado o quadro negro, onde eram relacionados: o nome do idoso em acompanhamento pela equipe, o nome do cuidador principal, endereço e telefone de contato, os nomes dos profissionais envolvidos no atendimento e a data da visita agendada. As visitas eram realizadas, na sua maioria, sem o uso de transporte. Em sendo necessário uso de condução, havia a possibilidade de realizar seu agendamento semanal, com base nos registros do quadro negro.

Foram estabelecidas planilhas de atendimento pelas quais os pacientes eram acompanhados na sua evolução e desfecho, assim como eram anotados aspectos relevantes desses e/ou de seus cuidados.

\section{Educação em serviço}

A educação em serviço ocorreu durante as reuniões semanais, na discussão de casos, por meio de leitura de bibliografias que eram trazidas pelos membros da equipe e estagiários, na visita a serviços de atendimento domiciliar e por meio de palestras.

A presença de alunos dos cursos de Graduação em Enfermagem foi fomentadora desse processo de educação permanente, abrindo espaços de discussão teórico-prática e integrando alunos e profissionais.

\section{Aspectos demográficos, sociais e de saúde dos idosos}

Foram cadastrados no projeto, 25 idosos, sendo 18 mulheres (86\%) e 7 homens (33\%). Receberam algum tipo de atendimento pela equipe 21 idosos e 4 não foram avaliados. Identificou-se que os idosos se concentravam nas faixas etárias entre 75 e 84 anos.

A maioria deles morava no bairro próximo à Unidade. As moradias foram construídas na década de 50 , caracterizandose por apartamentos em edifícios, de, no máximo, 3 andares, sem elevador e sistema de interfonia, sobrados geminados e casas. O tipo de moradia ficou dividido: 11 idosos moravam em apartamento (52\%) e 10, em casas ou sobrados (48\%).

Nos apartamentos, o acesso entre os andares era feito por escadas, sem corrimão, com degraus estreitos e escorregadios, de acabamento em cimento queimado. A iluminação, durante o dia, nos apartamentos e nos corredores de acesso era boa. Como não existia sistema de interfonia e de bloqueio das portas externas, o acesso aos apartamentos se dava quando o morador descia e abria a porta do prédio. Nos sobrados, o idoso acamado ficava no andar térreo, em um lugar disposto especialmente para ele. 
Quanto à existência de um cômodo da casa só para o doente e a presença de cuidador, 17 (81\%) possuíam cômodo só para si, sendo que o cuidador primário era um familiar que morava no mesmo domicílio. Apenas uma idosa morava sozinha e recebia os cuidados de um familiar que morava ao lado da casa.

Foi possível identificar que a família se envolvia cotidianamente com os cuidados da casa e do idoso acamado. Assumia as atividades de cuidados pessoais, de higiene, vestuário, alimentação, administração de medicamentos, troca de curativos, mobilização no leito, deslocamento do leito até a poltrona e, em alguns casos, caminhar, e haver exposição ao sol. Em apenas uma família, um profissional contratado, diariamente, em dois turnos, realizava os cuidados de higiene, curativo e caminhada.

As dificuldades na aquisição de materiais e equipamentos, bem como as adaptações do ambiente apareceram em diferentes aspectos. Constatou-se que as famílias se mobilizavam para a reorganização dos espaços físicos na casa, de forma que o idoso tivesse um cômodo só para si, mas o espaço reduzido entre a cama e o mobiliário, quartos e banheiros pequenos dificultavam a saída da cama, bem como a utilização de cadeiras de rodas. Outra questão importante identificada foi a criatividade dos cuidadores para prover tecnologias, de forma improvisada, que auxiliassem no cuidado prestado.

O deslocamento dos idosos em caso de atendimento na Unidade de Saúde, era difícil para a maioria deles, devido às barreiras arquitetônicas da moradia, das condições de acamado, bem como pelos aspectos financeiros envolvidos na contratação de um transporte privado, tendo em vista que a Secretaria Municipal da Saúde não dispunha de transporte público para remoções sociais.

Entre as atividades instrumentais de enfermagem mais requeridas para assistência dos idosos participantes do estudo estavam as relacionadas às seqüelas de doenças crônicas, não transmissíveis, após a alta hospitalar, tais como: cuidados com sondas enterais e vesicais, cuidados com traqueostomia, curativo de feridas cirúrgicas, retirada de pontos, orientação e cuidado com lesões de pele causadas por úlceras de pressão, entre outros. Também a enfermagem era solicitada para sanar dúvidas relativas à organização do ambiente doméstico para o cuidado domiciliar e para apoio a cuidadores, tendo em vista o desconhecimento e a ansiedade gerada na sua nova função. Na fisioterapia, o cuidado estava centrado na movimentação do acamado, no deslocamento da cama para a cadeira, na postura do cuidador para evitar lesões posturais durante o cuidado. Na fonoaudiologia baseava-se no diagnóstico e nas orientações mínimas ao cuidador domiciliar. Também havia a necessidade dos familiares em sanar dúvidas relativas à organização do ambiente doméstico, apoio emocional e vínculo, haja vista a ansiedade gerada pela função de cuidar em casa.

Pôde-se constatar que a família era capaz de suprir demandas diárias de cuidado, quando apoiada pelos profissi- onais de saúde; a esses cabia orientar e oferecer suporte a idosos e cuidadores, para que o cuidado acontecesse de forma tranquila e com menor desgaste para ambos, de forma que a integração dos envolvidos no processo trouxesse benefício ao doente e a sua saúde.

Em geral, as famílias se sentiam sozinhas e cansadas, na árdua tarefa de cuidar do doente acamado. Quando esta tarefa cabia a poucas pessoas, identificou-se que o cansaço era maior, pois cuidar de doentes em casa é tarefa que envolve uma rede de apoio que nem sempre existe, ou a família desconhece.

As dificuldades geradoras de ansiedade na família estavam relacionadas, principalmente, aos cuidados com as feridas e com sondas para alimentação.

Os custos dos tratamentos, aliados ao desconhecimento de parte dos cuidadores leigos do que seria uma possível complicação do quadro de saúde, causavam insegurança e tensão. Foi identificado que as famílias tinham necessidade de receber informações, de compreender o que estava acontecendo e de como deveriam agir na situação evidenciada.

\section{RESULTADOS E DISCUSSÃO}

\section{Aspectos relevantes evidenciados no desenvolvimento da assistência domiciliar}

A faixa etária dos idosos assistidos vem ao encontro dos dados do IBGE ${ }^{(4)}$ que indicam ter sido na idade de 80 anos ou mais que houve o maior índice de crescimento entre as pessoas idosas (2,4 milhões), em 2005. Também informam que nessa faixa etária as pessoas possuem maior incidência de doenças crônicas, piores capacidades funcionais, menor autonomia, e, consequentemente, exigem maior atenção da família e da sociedade, e, no que se refere ao sexo, nessa faixa etária há uma razão de 100 mulheres para cada 62 homens.

O número de mulheres acima de 60 anos, se comparado com o de homens é maior há muito tempo, sendo que a expectativa de vida das mulheres, ao nascer, é superior à dos homens, em torno de 4 anos. Cada vez mais, no Brasil, tem sido possível observar uma feminilização do envelhecimento ${ }^{(5)}$.

Apesar de este estudo apontar somente uma idosa morando sozinha, constata-se que é na Região Sul do Brasil que são encontradas $47,7 \%$ das famílias caracterizadas como sendo do tipo ninho vazio (casais sem filhos ou pessoas morando sozinhas). Já na Região Norte e Nordeste do País, a maioria dos idosos coabita com seus familiares, numa ordem de $70,5 \%$ e $68,3 \%{ }^{(4)}$.

Tem sido descrita a sobrecarga dos cuidadores primários com as atividades diárias de cuidado ao acamado, relatando seu sofrimento, mudanças no seu estilo de vida, despesas com transporte e a dependência do acamado ao cui- 
dador $^{(17-18)}$. O relato de insegurança, necessidade de receber informações e instrução quanto ao cuidado no domicílio também foram descritos em estudos brasileiros ${ }^{(17,19)}$.

$\mathrm{Na}$ velhice estão aumentados os riscos de doença, invalidez, viuvez, isolamento e de propensão para a morte. Com a passagem do tempo é impossível evitar o desgaste do corpo, sendo que à medida que a idade avança em anos o adoecimento ocorre com mais facilidade e a recuperação é mais lenta. Em geral as doenças nos idosos são crônicas e múltiplas, exigindo intervenções contínuas de equipes multidisciplinares. Lidar com essas questões faz parte do arsenal de habilidades que cada profissional ou equipe devem estar aptos a desenvolver, quando se propõem a cuidar de idosos.

Entretanto, o serviço de saúde, por não estar organizado a pleno, teve dificuldade em responder às demandas dos idosos vinculados ao projeto. Esta dificuldade ficou evidenciada nas necessidades de transporte do acamado até a Unidade de Saúde ou hospital, de avaliação por profissional médico, no domicílio, nos casos de agravamento das suas condições de saúde, de nutricionista, para ajustes de dieta e acompanhamento nutricional e de assistente social, para encaminhamento de necessidades sociais e de formação de parcerias com Instituições, sem falar no despreparo da equipe em lidar com casos de negligência de cuidados ao idoso.

Ao propor um projeto-piloto de atendimento domiciliar a equipe tinha a intenção de apoiar famílias no cuidado ao idoso, de forma que diminuíssem as complicações e as seqüelas instaladas, pudesse ser implementado um espaço educativo de trocas de saberes, que diminuíssem ansiedades, respeitando-se as crenças, os valores e as práticas que os cuidadores já desenvolviam, de forma a integrar o cuidado cultural e o profissional, reforçando o espaço de cuidado da família.

Desta forma influenciaram o fortalecimento do projeto determinados aspectos, como:

- O reconhecimento da grande necessidade e da importância dessa modalidade de assistência, para idosos, cuidadores, e familiares que necessitam sanar dúvidas, dividir preocupações e realizar planos de cuidados;

- A ampliação do leque de competências e trocas de saberes entre a equipe e família;

- O fato de o projeto ter iniciado sem a equipe completa e por iniciativa do corpo de enfermagem da Unidade;

- A motivação dos profissionais envolvidos, rompendo com o modelo de atenção, realizado somente no espaço da Unidade de Saúde;

- A busca pelo fortalecimento de intersetorialidade dos serviços públicos;

- A riqueza da experiência para os profissionais envolvidos, tanto nas relações interpessoais, técnico-assisten- cial e de estabelecimento de parcerias, destacando a grande responsabilidade de profissionais e cuidadores em sua atuação no domicílio;

- O início de um programa de educação permanente para os profissionais do projeto;

- A qualificação e ampliação das possibilidades de atendimento em decorrência da parceria com as Escolas de Enfermagem, no desenvolvimento de estágios curriculares;

- A elaboração, pela equipe, de instrumentos administrativos que qualificaram e possibilitaram o atendimento;

- A melhora que o trabalho coletivo proporcionou à relação das famílias com o serviço, à qualidade de vida aos acamados e à segurança e autoconfiança dos cuidadores.

Contudo, nem todos os aspectos evidenciados foram tão contributivos como os anteriormente descritos. Constatou-se fragilidade relacionada à:

- Ausência de profissionais na equipe: médico, nutricionista e assistente social;

- Morosidade na resposta à necessidade de avaliação médica no domicilio;

- Dificuldade na obtenção de transporte social para remoção do idoso aos Serviços de Saúde;

- Ausência de documento que estabelecesse a responsabilidade do cuidador e os limites da atuação da equipe;

- Sub-registros nos prontuários, sem um padrão único cada profissional o fazendo conforme a especificidade da sua atuação ou profissão;

- Ausência de instrumento padronizado que caracterizasse os atendimentos e o ambiente;

- Dificuldade dos profissionais envolvidos, em conciliar suas atividades, na Unidade de Saúde com a necessidade de visita domiciliar, mais que uma vez por semana e, principalmente, quando aumentaram o número de famílias a serem atendidas pelas equipes;

- A dependência do desenvolvimento do projeto quase que exclusivamente dos profissionais envolvidos, com pouca participação dos demais;

- A necessidade de instrumentos, materiais e equipamentos que pudessem ampliar e qualificar a assistência prestada (prontuário domiciliar, sondas, aliviadores de pressão, insumos para curativos, camas hospitalares, nebulizadores, cadeira de rodas, andadores, muletas, aspiradores, jogos, entre outros);

- A falta de parcerias com voluntários para atividades com idosos e de suporte para cuidadores nas Atividades de Vida Diárias e Instrumentais. Esta modalidade de atendimento, embora não tenha sido realizada, foi pensada, de forma que voluntários pudessem acompanhar idosos, realizando as atividades: leitura, jogos, troca de experiências, 
trabalhos manuais, entre outras, bem como permanecer no domicílio enquanto o cuidador realizava cuidados da casa, visitava a Unidade de Saúde ou realizava atividades instrumentais de vida diária.

\section{CONSIDERAÇÕES FINAIS}

Acredita-se que, com a realização deste estudo foi possível conhecer fatores relevantes sobre a realidade demográfica, social e de saúde dos idosos participantes do projeto - piloto, gerando informações fundamentais para a sua reestruturação.

Os idosos assistidos eram, em sua maioria, mulheres, predominantemente na faixa etária entre 75 a 84 anos moradores do entorno da Unidade de Saúde. O familiar era o cuidador principal que assumia todas as funções de cuidado da casa e do idoso acamado. Os principais motivos para solicitação de atendimento domiciliar eram as doenças crônicas não transmissíveis e suas seqüelas. As dificuldades geradoras de ansiedade na família estavam relacionadas, principalmente aos cuidados com feridas, sondas de alimentação e na obtenção de transporte para deslocamento do acamado para os Serviços de Saúde. Constatou-se que as famílias eram capazes de assumir demandas diárias de cuidado, quando apoiadas pelos profissionais de saúde.

Notou-se a influência, ora fortalecedora e ora fragilizadora, na intervenção de saúde realizada por meio do projeto-piloto de assistência domiciliar a idosos acamados.

A equipe tinha a intenção de apoiar famílias no cuidado ao idoso, diminuindo complicações e seqüelas instaladas, criando espaços educativos de trocas de saberes, minimizando ansiedades, com reforço dos espaços de cuidado da família. Para os profissionais envolvidos no projeto, identificou-se que a experiência foi enriquecedora, bem como para os cuidadores, destacando-se a grande responsabilidade assumida por ambas as partes na tarefa de cuidar, no domicílio. O trabalho coletivo melhorou a relação das famílias com a Unidade de Saúde e proporcionou qualidade de vida aos idosos, segurança e autoconfiança aos cuidadores.

É relevante mencionar que foi um terreno frutífero para o estabelecimento de parcerias entre Universidades e a Unidade de Saúde, promovendo espaço de integração para a formação de recursos humanos, qualificação de profissionais e de processos de trabalho, além de incentivo à produção científica.

A avaliação do projeto-piloto apontou para a sua continuidade; entretanto, a revisão dos aspectos fragilizadores, registrados no seu desenvolvimento foi essencial para a continuidade deste, fazendo-se necessários ajustes na organização para que se ampliassem os espaços de assis- tência e a qualidade do que era ofertado à população. Embora não fosse possível descrever a melhoria individual do quadro clínico dos pacientes atendidos, tendo em vista a utilização de fontes secundárias, identificou-se a melhora das condições de higiene, de mobilidade do paciente, de reorganização de espaços e de aspectos psicológicos das famílias que se sentiram apoiadas e mais seguras.

Ressalta-se que apesar do forte enfoque instrumental e normativo, a enfermagem esteve presente na maioria dos atendimentos e que o acompanhamento da fisioterapia e da fonoaudiologia qualificaram a atenção prestada, ampliando-se o espectro de ação das famílias cuidadoras, já que os casos atendidos, em sua maioria, eram de pessoas com problemas osteoarticulares e neurológicos.

A reflexão das autoras sobre este fato repousa em elementos como a imaturidade da discussão dos processos de trabalho pelo pessoal da enfermagem e o despreparo da Unidade de Saúde para apoiar as equipes, sob vários aspectos.

Desde sua criação o atendimento domiciliar tinha como principal meta romper com o cardápio pronto. Os profissionais, apesar de inseguros, tinham o desejo da mudança e buscaram arduamente um cuidado humanizado e cidadão, mas para isso precisaram romper com as estruturas rígidas que existia dentro deles e na Unidade.

A partir da avaliação dessa experiência, a equipe de saúde do PADA/IAPI vem criando um corpo de trabalho em busca do cuidado integral, de forma interdisciplinar e intersetorial, pautado pela soma de cuidados pactuados e articulados, e em resposta ao esforço árduo de comprometer-se cada vez mais com a vida e por um cuidado éticopolítico em saúde.

Os desafios impostos pelo comprometimento dos profissionais, no trabalho vivo de saúde, exigiram da equipe a busca de novas estratégias de organização do trabalho. Essas estratégias deveriam romper com o modelo biológico e médico centrado, restrito à área física da Unidade. Esse compromisso cidadão do cuidado evidenciou que somente a técnica era insuficiente para dar conta do direito à qualidade da vida e da morte digna. As práticas educativas internas à equipe, aliadas ao trabalho vivo em ato, favoreceram a conciliação da ética e da técnica, diminuindo a fragmentação e a dicotomização do cuidado dos cuidadores profissionais e leigos.

As autoras permitem-se destacar por fim que a experiência aqui relatada demonstra a reação dos atores em saúde à ação política de reconhecimento da vida pulsante em sua área de abrangência incorporando mesmo de forma insipiente elementos constituintes do cuidado integral em saúde. 


\section{REFERÊNCIAS}

1. Brasil. Ministério da Saúde. Secretaria de Assistência à Saúde. Guia operacional e portarias relacionadas. Brasília; 2002.

2. Silveira TM, Caldas CP, Carneiro TF. Cuidando de idosos altamente dependentes na comunidade: um estudo sobre cuidadores familiares principais. Cad Saúde Pública. 2006;22(8): 1629-38.

3. Püschel VAA, Ide CAC, Chaves EC. Modelos clínicos e psicossocial de atenção ao indivíduo e à família na assistência domiciliar: bases conceituais. Rev Esc Enferm USP. 2006;40(2):261-8.

4. Instituto Brasileiro de Geografia e Estatística (IBGE). Síntese de indicadores sociais 2006. Rio de Janeiro; 2006.

5. Organização Pan Americana de Saúde (OPAS). Indicadores básicos para a saúde no Brasil: conceitos e aplicações. Brasília; 2008.

6. Brasil. Ministério da Saúde. DATASUS. Sistema de Informações Epidemiológicas e Morbidade [texto na Internet]. [citado 2008 maio 25]. Disponível em: http://tabnet.datasus.gov.br

7. Rehem TCMSB, Trad LAB. Assistência domiciliar em saúde: subsídios para um projeto de atenção básica brasileira. Cienc Saúde Coletiva. 2005;10 Supl:231-42.

8. Silva KL, Sena R, Leite JCA, Seixas CT, Gonçalves AM. Internação domiciliar no Sistema Único de Saúde. Rev Saúde Pública. 2005;39(3):391-7.

9. Fabrício SCC, Wehbe G, Nassur FB, Andrade JI. Assistência domiciliar: a experiência de um hospital privado do interior paulista. Rev Lat Am Enferm. 2004;12(5):721-6.

10. Lahan CF, Silva LA. O cuidador. In: Jacob Filho W, editor. Avaliação global do idoso: manual da Liga Gamia. São Paulo: Atheneu; 2005. p. 171-80.
11. Porto Alegre. Secretaria Municipal da Saúde. Centro de Saúde IAPI. Projeto de Atendimento Domiciliar a Pacientes Acamados. Porto Alegre; 2002.

12. Hartz ZMA. Avaliação em saúde: dos modelos conceituais à prática na análise da implantação de programas. Rio de Janeiro: FIOCRUZ; 1997.

13. Conselho Nacional de Saúde. Resolução n. 196, de 10 de outubro de 1996. Dispõe sobre diretrizes e normas regulamentadoras de pesquisa envolvendo seres humanos. Bioética. 1996;4(2 Supl):15-25.

14. Freitas IB, Marques GQ, Ribeiro A, Boggio E. Caracterização de idosos de uma área descrita de uma unidade básica de saúde: níveis de dependência e tendências socioeconômicas. Saúde (Porto Alegre). 2004;1(1):65-70.

15. Porto Alegre. Secretaria Municipal da Saúde. Centro de Saúde IAPI. Relatório de avaliação do projeto-piloto de assistência domiciliar a idosos acamados. Porto Alegre; 2004.

16. Porto Alegre. Secretaria Municipal da Saúde. Centro de Saúde IAPI. Protocolo de procedimentos em nível de atenção primária para o atendimento domiciliar ao idoso acamado. Porto Alegre; 2002.

17. Diogo MJD'E, Ceolim MF, Cintra FA. Orientações para idosas que cuidam de idosos no domicílio: relato de experiência. Rev Esc Enferm USP. 2005;39(1):97-102.

18. Veras RP, Caldas CP, Dantas SB, Sancho LG, Sicsú B, Motta LB, et al. Avaliação dos gastos com o cuidado do idoso com demência. Rev Psiquiatr Clín (São Paulo). 2007;34(1):5-12.

19. Maffioletti VLR, Loyola CMD, Nigri F. Os sentidos e destinos do cuidar na preparação dos cuidadores de idosos. Ciênc Saúde Coletiva. 2006;11(4):1085-92. 